Service social

\title{
Les grands systèmes. Modèle de protection, modèle de justice et les perspectives d'avenir
}

\section{Oscar D’Amours}

Volume 47, numéro 3-4, 1998

Enfance : enjeux et réalités 2000

URI : https://id.erudit.org/iderudit/706794ar

DOI : https://doi.org/10.7202/706794ar

Aller au sommaire du numéro

Éditeur(s)

École de service social de l'Université Laval

ISSN

1708-1734 (numérique)

Découvrir la revue

Citer cet article

D’Amours, O. (1998). Les grands systèmes. Modèle de protection, modèle de justice et les perspectives d'avenir. Service social, 47(3-4), 15-40.

https://doi.org/10.7202/706794ar
Résumé de l'article

En matière d'évolution du droit des mineurs, deux grands systèmes se sont développés. Ainsi, le système de protection, inspiré de la doctrine parens patriøe, considère que l'enfant n'est pas responsable de ses actes, mais victime des circonstances : il ne faut donc pas le punir, mais le protéger. Le système de justice, inspiré de la nécessité d'offrir au mineur un « due process of law », recentre le débat sur l'acte délictueux et accorde une grande importance aux garanties procédurales.

Pour l'auteur, ni l'un ni l'autre de ces deux systèmes n'apporte de réponse à la délinquance des jeunes. Il faut chercher une nouvelle voie. 


\section{ARTICLES}

\section{Les grands systèmes Modèle de protection, modèle de justice et les perspectives d'avenir}

Oscar D'AMOURS

En matière d'évolution du droit des mineurs, deux grands systèmes se sont développés. Ainsi, le système de protection, inspiré de la doctrine parens patriæ, considère que l'enfant n'est pas responsable de ses actes, mais victime des circonstances: il ne faut donc pas le punir, mais le protéger. Le système de justice, inspiré de la nécessité d'offrir au mineur un "due process of law », recentre le débat sur l'acte délictueux et accorde une grande importance aux garanties procédurales.

Pour l'auteur, ni l'un ni l'autre de ces deux systèmes n'apporte de réponse à la délinquance des jeunes. II faut chercher une nouvelle voie.

Regarding the evolution of juvenile law, two major systems have taken place: the system of protection, inspired by the doctrine of parens patriæ, considers that the child is not responsible for his acts, but is the victim of circumstances. He must therefore be protected rather than punished. The justice model, inspired by the need to provide the minor with a due process of law, refocusses the debate on the offence and places major emphasis on the guarantees of the proceedings.

For the author, neither of those systems provides an answer to juvenile delinquency. We have to look for a new way. 


\section{INTRODUCTION}

La vie quotidienne d'un enfant se déroule toujours dans un environnement que le temps a façonné (Winterdyk, 1997, p. 329). Ainsi, malgré tous les efforts auxquels les organisations internationales ont consenti depuis de nombreuses années, il nous faut malheureusement déplorer que l'environnement de beaucoup d'enfants mineurs, encore aujourd'hui, ne comporte même pas un minimum de mesures de protection. Certains d'entre eux ne connaissent pas le minimum vital, d'autres sont l'objet de commerce, d'exploitation ou d'abus de la part de ceux ou celles qui ont pourtant mission de les protéger. On doit le reconnaître, c'est la plupart du temps l'endroit où un enfant vit qui détermine encore aujourd'hui le type de protection dont il est l'objet. L'enfant mineur est avant tout tributaire de ce que les adultes mettent à sa disposition et de la perception que ceux-ci ont de lui : ainsi l'enfant sera-t-il considéré comme un sujet de droit ou un objet de droit.

Le contexte économique, les valeurs sociales et les institutions sont les principales variables qui déterminent l'importance qu'on accorde à cet enfant. Celui-ci pourra-t-il espérer que des institutions substituts agiront en lieu et place de ses parents, lorsque ces derniers ne seront pas en mesure de lui assurer une protection adéquate? Et s'il contrevient à une loi, pourra-t-il bénéficier d'une approche qui tienne compte à la fois de ses droits, de ses besoins et de son intérêt ou sera-t-il au contraire soumis à une approche arbitraire, voire discriminatoire, sans qu'il puisse jouir d'une véritable protection sociale?

Depuis une centaine d'années de nombreuses expériences ont été menées un peu partout dans le monde pour répondre à ces questions. Au cours des décennies, c'était souvent un événement et la réaction de la population à l'événement touchant un enfant qui enclenchaient une action pouvant apporter la réponse adéquate aux besoins identifiés. Avec le temps, les mécanismes d'identification, de qualification et de solution des problèmes relatifs à l'enfant ont changé. De tels événements sont désormais fortement médiatisés, les réactions sont rapides et les solutions proposées ne sont pas toujours une réponse aux besoins réels 
des enfants mineurs, mais cherchent plutôt à satisfaire les besoins d'une opinion publique nerveuse et volatile.

Au fil des ans, deux grands systèmes de justice à l'endroit des mineurs ont eu droit de cité : le modèle de protection, qui met l'accent sur la réhabilitation et les besoins du délinquant, et le modèle de justice, qui privilégie le processus légal et la responsabilisation du mineur ${ }^{1}$.

Dans cet exposé, nous allons analyser ces deux modèles d'intervention afin d'en saisir les tenants et les aboutissants et d'en faire ressortir les différences caractéristiques. Pour chacun de ces deux modèles, nous allons rappeler le contexte qui l'a fait naître, décrire son contenu et faire part des critiques dont il fut l'objet. Puis nous indiquerons certaines perspectives d'avenir sur un nouveau modèle d'intervention à privilégier.

\section{LE MODĖLE DE PROTECTION}

\section{Le contexte}

Jusqu'à l'ère moderne, on peut dire que l'enfant mineur était habituellement pris en charge par sa famille et, au besoin, par la famille élargie. Dans la société préindustrielle, c'est donc la famille qui demeurait l'institution servant à la fois de lieu de socialisation, d'éducation et de consommation. Avec la transformation progressive, au début du $19^{\mathrm{e}}$ siècle, de la société en une structure qui s'industrialise, les tâches de la famille elle-même se trouvent redistribuées et un nouveau mode de vie familiale voit le jour (Empey, Stafford et Hay, 1999, p. 50 et s.).

Le rôle de l'État à l'endroit des enfants mineurs subira également une transformation. Ainsi, sous l'influence de groupes de pression, l'État sera amené progressivement à favoriser la mise en place de ressources plus adéquates afin d'assurer la protection de ses membres, notamment celle des plus faibles d'entre eux, les enfants.

1 Juvenile Justice Models, Australian Law Reform Commission, report no. 84, 1997 p. 476. 
Pour justifier son intervention au sein de la famille, l'État s'inspire alors de la doctrine du parens patriæ, qu'il importe d'Angleterre tout en l'adaptant au contexte nord-américain. On en arrive alors à considérer l'enfant mineur non plus comme étant responsable de ses actes, mais plutôt comme une victime des circonstances et du caractère inadéquat de son milieu. On ne doit donc pas le punir pour des gestes qu'il a certes posés, mais dont il n'était pas responsable; il faut plutôt le protéger contre des facteurs qui lui sont extérieurs. Selon ce modèle, l'intervention doit se centrer non pas sur la punition à infliger, mais sur la satisfaction des besoins spécifiques du mineur.

On procède alors à la création de refuges pour enfants mineurs en les considérant un peu comme la panacée à tous les maux vécus par les enfants et les adolescents, que ceux-ci soient en danger ou délinquants. L'expérience démontrera par la suite que la mise en place de ces refuges n'a pas donné les résultats escomptés. On reconnaissait cependant qu'elle avait eu comme effet positif d'assurer désormais l'hébergement des mineurs dans des endroits distincts de ceux des adultes criminels (Empey et collab., 1999, p. 53).

La remise en question de ces refuges a amené la mise en place de nouvelles institutions, soit les écoles de réforme et de l'industrie, qui privilégiaient une philosophie moins punitive que celle qui existait dans les refuges. Mais ces nouvelles institutions n'échappèrent pas, elles non plus, aux critiques adressées aux anciens refuges.

Ces diverses expériences ont progressivement amené la société à reconnaître que les mineurs avaient des besoins qui leur étaient propres et qu'il fallait alors faire appel pour eux à un modèle de traitement différent de celui des adultes. La société découvrait ainsi le concept moderne de l'enfance et reconnaissait que l'enfant mineur était immature, en recherche d'autonomie et incapable d'apprécier les conséquences de son comportement (Empey et collab., 1999, p. 57). Accepter une telle orientation, c'était renoncer à maintenir le même traitement pour les enfants que celui en vigueur dans le système adulte. II nous suffisait de franchir ce pas pour que le système de justice pour mineurs voie le jour. 
C'est à Chicago, en 1899, qu'on a vu apparaître la première cour de justice pour les mineurs (Empey et collab., 1999, p. 58). Elle visait tout d'abord à décriminaliser la conduite délinquante des mineurs et à les aider non pas à partir de l'examen de l'infraction commise, mais à partir de celui de leurs besoins spécifiques.

Cette bienveillance à l'égard des mineurs se manifestera donc tout autant à l'endroit de l'enfant en danger qu'à l'endroit de celui qui a commis une infraction. Les deux catégories de mineurs se retrouvaient dans une situation analogue qui requérait de la part des adultes une aide appropriée. C'est ainsi qu'est né le modèle de protection qui s'applique alors aussi bien aux enfants en danger qu'aux délinquants.

\section{Description}

En conformité avec les théories de l'époque, les législateurs vont s'écarter de l'école classique de droit pénal (Empey et collab., 1999 , p. 63) pour adopter une formule nouvelle concernant les droits des mineurs. Cette formule s'appuie sur la prémisse que les causes de la délinquance chez le mineur sont d'origine sociale et que celui-ci exerce peu de contrôle sur ces causes (Trépanier et Tulkens, 1995, p. 61 et s.). Comme le souligne Jean Zermatten, « l'enfant est considéré comme une victime de son milieu et des circonstances et les soins à lui apporter sont déterminés non pas par son comportement, mais par l'examen de sa situation personnelle » (1994, p. 171).

C'est ainsi que le modèle de protection se trouvait à privilégier une intervention à partir des besoins du mineur (Trépanier, 1989, p. 566) et non plus en fonction de l'acte qu'il avait commis, comme le spécifiait le droit pénal classique.

Dans ce contexte, l'État devient en quelque sorte un « superparent » dont l'objectif est désormais de protéger l'ordre social en intervenant sur les causes de la délinquance et en privilégiant non pas la punition, mais la réhabilitation du mineur délinquant (Empey et collab., 1999, p. 325 et s.).

Jusqu'à la fin des années 1980, bon nombre de législations nationales vont adopter ce modèle d'intervention qui s'éloigne du 
concept de punition-rétribution-répression afin d'épouser un modèle élaboré autour des concepts d'aide, d'assistance et de prise en charge. C'est ce modèle que Françoise Tulkens (1996) a défini comme étant le modèle protectionnel (p. 198).

Dans ce modèle protectionnel, c'est la personne du mineur délinquant qui constitue la mesure de toute intervention. La question n'est plus de rechercher si un mineur peut être responsable de son comportement criminel, mais plutôt comment on peut mieux le traiter pour favoriser sa socialisation et éviter sa criminalisation. L'important est de procéder à une définition précise des besoins du mineur afin de lui offrir une aide adéquate.

Si la famille ne peut pas jouer ce rôle d'aide et d'assistance, l'État doit intervenir. II revient alors au juge d'assurer cette définition des besoins du mineur et de déterminer les causes de son comportement criminel. À cet égard, il pourra faire appel à des spécialistes qui l'éclaireront sur l'aide appropriée à fournir au mineur. C'est alors qu'entrent en scène les agents de probation et les travailleurs sociaux dont le rôle sera désormais d'assumer la prise en charge des mineurs déviants.

Le modèle de protection rompt de cette façon avec la philosophie punitive de la justice criminelle pour les adultes et l'on met fin à la recherche d'une proportionnalité de la peine avec la gravité de l'infraction. L'accent est plutôt mis sur le traitement, ce qui amènera souvent le juge à ordonner une prise en charge du mineur suffisamment longue pour que ce traitement puisse avoir toute son efficacité. Aussi ne faut-il pas se surprendre si l'on procédait alors à des placements et si l'on ordonnait d'autres mesures restrictives de liberté non seulement à l'endroit de mineurs impliqués dans un délit, mais également comme moyens de prévention, dès qu'apparaissaient les premiers signes d'un comportement déviant.

Dans le modèle de protection, le juge joue un rôle déterminant et exerce une large discrétion. En effet, une fois saisi de la situation d'un mineur, il peut réviser, prolonger ou adapter les mesures selon l'évolution de la situation sans que nécessairement une autre situation de délit lui soit soumise. 
Après l'intervention de la Cour, il revient à l'agent de probation de prendre en charge le mineur concerné, de veiller à l'application de son plan de traitement et d'informer le juge de l'évolution de la situation.

\section{Critiques}

Il faut le reconnaître, ce modèle de protection, né du désir de soustraire le traitement des mineurs au régime réservé aux adultes criminels et de tenir compte de leurs besoins spécifiques, a fait l'objet de nombreuses critiques.

D'une part, le postulat de base établissant que le mineur est toujours victime de son milieu a été rapidement remis en question. De toute évidence, on ne pouvait pas toujours établir un lien de cause à effet entre le comportement délinquant d'un mineur et les caractéristiques de son milieu.

D'autre part, on déplorait, dans ce modèle de protection, l'absence de règles de droit claires. Cela donnait libre cours à des décisions qui pouvaient dépendre davantage des valeurs personnelles et subjectives des différents intervenants ou de critères moraux dont on peut se demander s'il revenait à la loi pénale de les imposer. On constatait par exemple que, dans certaines causes similaires, les décisions prises étaient souvent disparates et même parfois contradictoires.

II faut ajouter que dans le modèle de protection le juge assumait un rôle qui apparaissait flou et imprécis. De plus, lorsqu'il ordonnait une mesure d'aide à l'enfant d'une durée indéterminée, il se trouvait à abdiquer son rôle indispensable d'arbitre, en laissant désormais la discrétion judiciaire entre les mains de l'agent de probation ou de l'agent correctionnel. Car, dans les faits, c'est ce dernier qui accordait la libération du mineur, en mettant fin à son traitement (Empey et collab., 1999, p. 369).

L'efficacité de l'approche prônée par le modèle de protection pour prévenir la récidive chez les mineurs délinquants fut aussi largement contestée (Cullen et Egilbert, 1982, p. 170) et les méthodes utilisées pour la réhabilitation également remises en question. Comme le modèle de protection se préoccupait essentiellement 
des besoins du mineur délinquant et non pas de l'infraction que celui-ci avait commise, on pouvait se retrouver devant certaines interventions nettement abusives. Ainsi, un mineur ayant commis une infraction de peu de gravité pouvait se voir imposer, pour éviter la récidive, une mesure d'aide de longue durée en raison des besoins spécifiques qu'il présentait.

L'évolution du contexte sociétal a aussi fait apparaître d'autres lacunes au modèle de protection et suscité de nombreuses critiques qui se traduisirent par des revendications, notamment les revendications des cinq " $d$ ", selon l'expression de Françoise Tulkens: désinstitutionnalisation, déjudiciarisation, droits (octroyés, reconnus et défendus), diversion et décentralisation (Tulkens, 1996, p. 201).

On contestera enfin et surtout que ce modèle de protection puisse être appliqué aussi bien au mineur délinquant qu'à l'enfant ou à l'adolescent en danger par suite d'abus, de négligence ou d'abandon.

C'est pourquoi ce modèle de protection, largement répandu en Europe et en Amérique du Nord, a été graduellement remis en question et abandonné, notamment dans les pays de droit anglosaxon, à la suite d'un certain nombre de décisions de la Cour suprême des États-Unis durant les années 1966, 1967 et 1970.

\section{LE MODÈLE DE JUSTICE}

\section{Le contexte}

La seconde moitié du $20^{\mathrm{e}}$ siècle constitue une période au cours de laquelle on met un accent nouveau sur les droits de la personne. Cette orientation donnera naissance à une série d'importants instruments provenant d'organismes internationaux ${ }^{2}$ et à l'adoption de chartes pour protéger les droits individuels ${ }^{3}$. De nombreux pays adopteront également diverses lois visant à reconnaître des

2 Nous traiterons de ces instruments aux pages 29 et s.

3 À titre illustratif, voir la Charte des droits et libertés de la personne du Québec, L.R.Q., C. C-12 (1974) et la Charte des droits et libertés du Canada (1982). 
droits spécifiques à des personnes particulièrement vulnérables en raison de leur déficience mentale ou de leur âge.

Ce changement de contexte sociétal a amené de nombreux citoyens et organismes de défense des droits à dénoncer les abus que subissaient des mineurs qui ne bénéficiaient d'aucune protection procédurale. On déplorait notamment que certains d'entre eux se voient imposer des mesures d'une durée indéterminée pour des actes qui parfois ne constituaient même pas des infractions quand ils étaient accomplis par des adultes ${ }^{4}$. De plus, l'opinion publique était devenue davantage sensible et n'hésitait pas à remettre en question l'efficacité des méthodes de réhabilitation employées pour empêcher la récidive chez les mineurs et chez les adultes.

Ce nouveau contexte a amené graduellement la société à remettre en question la philosophie du modèle de protection qui visait essentiellement à répondre aux besoins d'un mineur au détriment des droits qu'elle avait de se protéger contre un comportement délinquant. De plus, on constatait une insuffisance flagrante des ressources pour assurer un traitement adéquat aux mineurs délinquants. On finit par en conclure que les mineurs ne recevaient ni traitement efficace ni sanction dissuasive pour contrer leur comportement délinquant (Hylton, 1994, p. 232 et s.).

Un autre élément important devait ébranler les bases du modèle de protection dans les années 1960 et 1970. En effet, la Cour suprême des États-Unis rendait alors trois décisions (Kent, Gault et Winship) qui remettaient fondamentalement en question ce modèle en ordonnant l'application d'un certain nombre de règles de procédure à l'endroit des mineurs. Ainsi, le mineur avait désormais droit à l'application du "due process of law », c'est-àdire qu'il avait droit à une audition, à la consultation d'un avocat et à la prise de connaissance des différents rapports que le juge possédait à son sujet (Kent) ${ }^{5}$.

${ }^{4}$ Prix Charles-Coderre 1993, L'adolescent et la Loi sur les jeunes contrevenants, Cowansville, Éd. Yvon Blais, p. 115.

5 383, US, 541, 1966. 
La Cour suprême précisait par la suite que le mineur non seulement droit avait à une audition et aux services d'un avocat, mais il avait le droit en plus de recevoir un avis d'audition, de connaître la nature des actes qu'on lui reprochait, d'interroger les témoins et de conserver le privilège de ne pas s'incriminer (Gault) ${ }^{6}$.

En 1970, la même cour déclare que les tribunaux pour mineurs ne sont pas nécessairement des cours criminelles. Elle proclame, par ailleurs, qu'en vertu de la Constitution américaine les mineurs, à l'instar des adultes, ont le droit strict d'exiger que le degré de preuve applicable dans les cas d'infraction criminelle soit établi hors de tout doute raisonnable (Winship) ${ }^{7}$.

À la suite de ces décisions, on distinguera de plus en plus clairement, surtout aux États-Unis, le traitement judiciaire des mineurs en danger (abus, négligence, etc.) de celui concernant les mineurs délinquants.

\section{Description}

C'est dans les années 1970 que s'élaborera progressivement le modèle de justice avec un accent mis désormais non plus sur les seuls besoins spécifiques du mineur délinquant, mais sur son acte délictueux. Dans ce modèle, le mineur doit assumer la responsabilité de ses choix et de ses gestes; la sanction qu'on lui impose doit être proportionnelle à la gravité du délit qu'il a perpétré. On doit également viser à assurer la protection de la société. De même, on verra à appliquer à l'endroit du mineur les principes suivants (Cullen et Egilbert, 1982, p. 125-126) auxquels ont droit les adultes en matière de justice pénale :

1- Délimiter les sentences pour qu'il y ait justice et éliminer la discrétion dans le système.

2- Imposer la sanction en fonction de l'infraction commise et non pas en vue de favoriser un plan de traitement individualisé : à cet égard, fixer le temps d'incarcération en fonction de la gravité de l'infraction

\footnotetext{
${ }^{6} 387$, US, 1, 1967.

${ }^{7}$ 397, US, 358, 1970.
} 
et non pas en fonction de la durée d'un plan de traitement.

3- Prévoir dans la loi la nature et la durée des sentences.

4- Réduire le temps d'incarcération.

5- Encadrer l'exercice de la discrétion judiciaire.

6- Abolir la libération conditionnelle.

7- Favoriser et augmenter les programmes volontaires de réhabilitation.

8- Reconnaître le droit de toute personne d'être incarcérée dans un environnement humain.

En ce qui concerne le mineur, le modèle de justice doit mettre en place une procédure assurant désormais le respect de ses droits. Ainsi, le mineur aura le droit d'être représenté par un avocat. Les décisions prises à son endroit le seront à partir de critères précis et devront être consignées afin de lui permettre d'en demander la révision ou d'en appeler. De plus, il a le droit d'être consulté et de participer à une consultation avec un conseiller et ses parents, si l'intérêt de ceux-ci n'est pas incompatible avec son propre intérêt. Enfin, la nature de la sentence doit tenir compte de l'acte délictueux du mineur et non pas de ses seuls besoins.

En somme, dans le modèle de justice, le mineur délinquant n'est plus perçu comme une innocente victime de son milieu, mais comme une personne responsable de ses choix et devant en assumer les conséquences. Quant à la Cour, elle ne doit intervenir que si l'infraction commise a été explicitement prévue dans une loi, sinon son intervention n'apparaît plus légitime.

En ce qui a trait à la protection de la société, on visera à l'assurer désormais en sanctionnant rigoureusement l'infraction commise par le mineur. Cela n'empêche nullement par ailleurs de tenir également compte des besoins personnels du jeune, mais dans les limites de la discrétion encadrée du magistrat. Un tel modèle de justice n'exclut pas les autres institutions sociales de l'aide à apporter au mineur délinquant, mais il limite et précise le caractère discrétionnaire de leurs interventions, ce qui a l'avantage de mieux définir et de départager de façon plus précise les fonctions respectives du système judiciaire et du milieu social. 


\section{Critiques}

La mise en place de ce modèle de justice a certes eu pour effet de redonner son caractère pénal à l'acte délictueux du mineur, mais le modèle a aussi précisé les droits reconnus et garantis au mineur lors des procédures. C'est ainsi que la reconnaissance explicite des droits du mineur et la mise en place de procédures en garantissant le respect allaient le protéger contre toute intervention non justifiée de l'État dans sa vie privée et contre toute restriction ou privation non motivée de sa liberté.

Par ailleurs, ceux qui avaient cru que le remplacement du modèle de protection par le modèle de justice aurait comme conséquence magique de mettre fin à la criminalité des mineurs faisaient une analyse simplifiée des vrais problèmes. On doit admettre, en effet, que le modèle de justice, tout comme celui de protection qui l'a précédé, laisse perdurer les disparités économiques et sociales qui favorisent souvent l'émergence de la délinquance : ce modèle a négligé de prendre en compte les mesures de prévention (Cullen et Egilbert, 1982, p. 133).

Pour les tenants du modèle de protection, le modèle de justice comporte une grave lacune dans la détermination de la mesure qu'il faut privilégier à l'endroit d'un délinquant mineur. Examinons, comme exemple, la situation de deux mineurs ayant commis des infractions. Le premier est accusé d'une infraction mineure, mais sa délinquance est bien connue et il aurait commis de nombreux autres délits dont la police n'est pas en mesure de faire la preuve. Quant à l'autre jeune, il est accusé d'une infraction grave, mais il en est à sa première infraction et on a tout lieu de croire qu'il ne récidivera pas. Si nous nous en tenons rigoureusement au principe de proportionnalité, on appliquera donc une mesure plus sévère au deuxième mineur, même s'il en est à une première infraction et qu'il n'y a pas de risque de récidive.

Le modèle de justice a fait aussi l'objet d'autres critiques qui sont davantage d'ordre pratique (Hackler, 1991, p. 205 et s.). Ainsi, la Cour, devant agir désormais dans un cadre plus formel, risque de porter moins d'attention aux situations plus particulières de chaque jeune délinquant et peut dispenser une justice à la chaîne comme cela se produit dans le système pénal pour adultes, en 
recourant par exemple pour le choix des mesures à une liste de tarification des diverses infractions. Dans un tel système, il n'y a pas de place pour les besoins spécifiques des individus et les mesures imposées risquent de ne plus avoir de portée éducative.

D'autre part, le système semble avoir favorisé chez certains procureurs le recours abusif à des arguments d'ordre technique. Cela est particulièrement déplorable à l'étape cruciale du choix de la mesure. On risque alors de choisir une mesure qui passe gravement à côté de l'intérêt du mineur.

Ajoutons enfin que le recours à une procédure formelle est toujours plus coûteux qu'une procédure informelle, qu'il exige un personnel plus nombreux et occasionne parfois de regrettables retards dans un traitement de la situation délinquante d'un mineur qui doit pourtant être contemporain au délit pour être signifiant et efficace.

À la lumière de ces critiques, faut-il revenir au modèle de protection ou encore maintenir, voire renforcer le modèle de justice? Nous sommes d'avis que, si l'on tient compte de l'évolution de la société et des sciences du comportement, il ne faut retenir ni l'un ni l'autre de ces modèles comme solution à la délinquance et cheminer plutôt vers un tout nouveau modèle que nous appellerons le modèle de justice réparatrice et que nous décrirons dans les perspectives d'avenir que nous allons maintenant tracer.

\section{LES PERSPECTIVES D'AVENIR}

La recherche d'une nouvelle voie pour le traitement des mineurs délinquants doit s'effectuer en prenant en compte un certain nombre de données tirées de l'expérience des dernières années.

II importe, dans un premier temps, de continuer à bien distinguer le traitement que l'on doit mettre en place pour le mineur en danger de celui que l'on devrait appliquer pour le traitement du mineur délinquant. Cette orientation est essentielle et elle a été amorcée au début des années 1970. L'expérience démontre, en effet, que le modèle de protection peut tout à fait convenir dans le traitement du mineur en danger, à la condition bien sûr qu'on assure à ce dernier les garanties légales de respect de ses droits. À cet égard, 
on constate que cette problématique de l'enfant en danger devient une cible de plus en plus importante dans de nombreux pays et qu'elle requiert souvent la plus grande partie de l'intervention des juges des mineurs.

Par ailleurs, l'expérience a également démontré qu'il ne serait pas pertinent de favoriser un retour au modèle de protection pour suppléer au modèle de justice dans le traitement du mineur délinquant. Pour Jean Zermatten (1994, p. 175), dès le début des années 1990 il ne fallait pas dépenser des énergies dans la recherche d'application d'un tel modèle. II apparaît donc irréaliste de s'orienter vers le modèle de protection pour le mineur délinquant. Bien sûr, la réhabilitation peut et doit toujours être poursuivie avec certains jeunes, mais elle ne peut constituer le seul objectif de l'intervention judiciaire. II faut lui ajouter aujourd'hui la protection de la société et la responsabilisation du mineur (Bala, 1994).

Intégrer les deux modèles de protection et de justice ne semble pas plus réaliste, car leur philosophie de base s'appuie sur des concepts qui s'opposent. Pour l'un, l'enfant est victime de son milieu, alors que pour l'autre le délinquant doit répondre de ses actes délictueux.

II ne faut pas davantage compter sur l'application d'un modèle de justice comme celui préconisé dans les années 1970. Celui-ci, en effet, n'a pas fait lui non plus la preuve qu'il pouvait enrayer la délinquance. Faut-il alors revenir pour les mineurs délinquants au régime réservé aux adultes? On sait que, depuis le début des années 1990, on assiste dans plusieurs pays ${ }^{8}$ à un durcissement des moyens mis en avant par la société pour enrayer la délinquance. Mais tous conviendront que ce serait alors faire fi des nombreux acquis positifs des cinquante dernières années en matière de traitement de la délinquance juvénile.

Ce sont là de précieuses données dont il nous faut tenir compte pour amorcer notre réflexion sur des perspectives d'avenir.

\footnotetext{
${ }^{8}$ Belgique, France, Canada, Angleterre, États-Unis.
} 


\section{Le contexte}

Depuis les années 1960, des organismes internationaux comme les Nations Unies et le Conseil de l'Europe ont élaboré un ensemble d'instruments relatifs à l'enfance et à la délinquance juvénile qui apportent un tout nouvel éclairage dans notre recherche de perspectives d'avenir.

En effet, de 1959 à 1996, les Nations Unies ont mis à la disposition de tous les pays pas moins de cinq instruments concernant les enfants mineurs. La nature de ces instruments (McCarney, 1997, p. 1-3) démontre l'importance que la communauté internationale accorde à l'enfant.

Déjà en 1959, les Nations Unies ont adopté Les Dix Principes de la déclaration des droits de l'enfant (résolution 1386 XIV), qui proclament en termes généraux les droits fondamentaux de l'enfant et décrivent ses besoins de base.

Le 29 novembre 1985, Les Règles de Beijing (R-40/33) concernant l'administration de la justice pour les mineurs précisent les garanties auxquelles tout enfant mineur, qu'il soit délinquant ou en danger, serait en droit de s'attendre devant les tribunaux. Ces règles prévoient des garanties procédurales pour les mineurs et elles indiquent les orientations à privilégier dans le choix des mesures les concernant. Elles optent clairement contre la privation de liberté, sauf lorsque le mineur est déclaré coupable d'une infraction grave. Elles encouragent enfin la mobilisation et la coopération du milieu pour qu'il collabore au traitement des mineurs.

La Convention relative aux droits de l'enfant, adoptée par la résolution 44/25 le 20 novembre 1989, enjoint à tous les États du monde d'assurer la protection des droits de l'enfant. Comme le souligne Willie McCarney (1997) :

La Convention devrait être considérée comme un point de repère majeur dans le domaine des droits de l'homme en général ainsi qu'en ce qui concerne les droits de l'enfant. Elle constitue une énumération complète des obligations envers l'enfant que les États sont tenus de reconnaître. Ces obligations peuvent être d'un 
caractère direct, par exemple assurer la scolarisation et la bonne administration de la justice des mineurs, ou bien indirect, permettant aux parents, la famille dans le sens plus large ou les gardiens d'accomplir leurs rôles et responsabilités en tant que gardiens et protecteurs. La Convention a été le premier instrument international à adopter une façon cohérente, en accord avec les droits de l'enfant, d'aborder la réglementation de la privation de liberté de mineurs (p. 2).

Le 14 décembre 1990, les Nations Unies adoptent un autre précieux instrument, soit Les principes directeurs de Riyad (résolution 44/112), pour la prévention de la délinquance juvénile. Ces principes fondamentaux mettent en évidence que la meilleure façon de prévenir le crime est de prévenir la délinquance juvénile; que cette action doit impliquer toute la société; que les jeunes doivent avoir un rôle actif de partenaires dans la société; que les programmes de prévention devraient être axés sur le bien-être des jeunes dès la petite enfance; que des programmes d'évaluation et de déjudiciarisation devraient être établis, concrétisés dans des textes législatifs et axés sur les besoins spécifiques; et, enfin, qu'on doit mettre en place des programmes communautaires de prévention.

Ces principes doivent s'appliquer dans le processus de socialisation tant dans la famille, à l'école que dans la communauté. De plus, leur réalisation ne serait possible qu'avec l'appui des médias, des politiques sociales et d'une législation appropriée.

Enfin, l'Assemblée générale des Nations Unies adopte, le 14 décembre 1990, Les règles de la Havane (résolution 45/113) pour la protection des mineurs privés de leur liberté. Ces règles, au nombre de 87 , s'appliquent autant pour le jeune délinquant que pour le mineur en danger et sont élaborées dans les " perspectives fondamentales » suivantes:

- l'incarcération est une mesure de dernier recours;

- le mineur ne peut être privé de liberté que pour des périodes fixes et en conformité avec les règles de Beijing;

- la privation de liberté doit être limitée à des cas d'exception, sans écarter la possibilité d'une libération 
anticipée, et elle ne peut être imposée que par une autorité judiciaire;

- les règles doivent être appliquées sans aucune discrimination;

- les règles doivent servir de référence à ceux qui participent à l'administration de la justice;

- les règles doivent être incorporées dans les législations nationales;

- les pouvoirs publics doivent assumer un rôle de sensibilisation du public et faciliter l'intégration des mineurs dans la communauté.

En plus de ces documents issus des Nations Unies, rappelons également les deux recommandations du Conseil de l'Europe, dont celle de 1987 (R 87-20) qui affirme que « le système pénal des mineurs doit continuer à se caractériser par son objectif d'éducation et d'insertion sociale. Elle [la résolution] porte l'accent sur les réponses alternatives que doit mettre en place la justice des mineurs » (Zermatten, 1994, p. 165).

Quant à la recommandation de 1988 (R 88-6) du même Conseil de l'Europe, elle souligne que les États doivent réduire l'écart entre les nationaux et les non-nationaux de façon à faciliter l'intégration de ceux-ci dans leur pays de résidence.

Le 25 janvier 1996, à Strasbourg, l'Assemblée parlementaire présente la Convention européenne sur l'exercice des droits des enfants, qui s'inscrit dans la promotion des droits des enfants, telle que préconisée par la Convention des Nations Unies de 1986. Elle se veut donc un instrument pour faciliter l'exercice des droits matériels des enfants en assurant des garanties procédurales qui peuvent être mises en œuvre par l'enfant ou par une autre personne au nom de l'enfant.

Notons également qu'en plus de ces diverses conventions nous disposons d'un modèle de loi sur la justice des mineurs publié dans la revue de l'Association internationale des magistrats de la jeunesse et de la famille (A.I.M.J.F., 1998, p. 7-24). 
Enfin, à l'ensemble de ces conventions, règles et modèles de loi se greffe une notion juridique, soit celle de l'intérêt de l'enfant (Fulchiron, 1997, p. 29 et s.), que les tribunaux ont interprétée au fil des années et des situations. En ce qui concerne le mineur délinquant, cette notion de l'intérêt de l'enfant doit être utilisée avec parcimonie, car comme l'indique Sophie Ballestrem (1997, p. 9), juge des mineurs de Munich :

L'idée générale du «meilleur intérêt de l'enfant », qui forme la base des interventions envers les enfants qui se comportent de manière délictueuse, échappe à toute définition légale précise et donne une grande mesure de pouvoir discrétionnaire aux juges et aux autres autorités. Elle manque de critères objectifs et ouvre la voie aux abus graves, aux dépens du mineur sous le prétexte de "ses meilleurs intérêts ». Par conséquent, pour sauvegarder les droits procéduraux de base que la convention ainsi que les règles de Beijing accordent aux enfants délinquants, il est important de faire une distinction plus nette entre les mineurs en question: le mineur en danger qui nuit à son développement éducatif par un comportement mal adapté mais non délictueux, et le mineur délinquant (le plus souvent en danger aussi), celui qui, en commettant l'acte, se rend compte des conditions de l'infraction.

L'ensemble de ces documents (conventions, chartes, etc.) constituent d'importants instruments mis à la disposition des États nationaux pour favoriser cette lente montée du droit de l'enfant mineur dans le monde. Ils nous fournissent également de précieuses balises dans notre recherche d'un nouveau modèle d'intervention auprès des délinquants mineurs.

\section{DESCRIPTION}

D'ores et déjà, nous devons retenir un certain nombre d'éléments constituants de ce nouveau modèle d'intervention auprès des délinquants mineurs. Ainsi, de nombreuses institutions seront désormais appelées à intervenir dans le domaine de la délinquance juvénile. On devra mieux définir et coordonner les fonctions respectives de ces divers intervenants. Et, dans une société où l'on retrouve désormais un partage complexe de responsabilités, il 
faudra reconnaître le rôle fondamental du magistrat pour trancher les différends qui peuvent survenir dans les interventions, et cela, dans le respect de la diversité des fonctions.

Certains acquis doivent également être maintenus :

- L'intervention du juge doit être prévue dans la loi.

- On doit maintenir des garanties procédurales pour tous les mineurs.

- L'approche législative en matière de délinquance juvénile et celle en matière de protection de l'enfance doivent être dissociées.

- Une révision judiciaire de toute décision sociale impliquant les droits du mineur doit être prévue.

- La possibilité de porter en appel toute décision judiciaire doit être accessible à l'enfant, aux parents et aux institutions sociales.

- Les principes élaborés dans la Convention des Nations Unies relative aux droits des enfants doivent être intégrés dans les législations nationales.

Le grand défi de la mise en place d'un nouveau modèle d'intervention auprès des mineurs délinquants pour les années 2000 réside dans l'inévitable antinomie de sa formule. II s'agit, en effet, de concilier un certain nombre de contradictions apparentes. Ainsi, ce modèle devra s'inscrire dans la continuité du changement, la diversité des solutions et l'unité d'action, dans une société nationale forcée de s'arrimer à des valeurs internationales, lesquelles doivent servir de base à des actions des communautés locales.

II importe au plus haut point de réaffirmer l'importance de la famille comme base du système de prévention du comportement déviant. C'est elle, en effet, qui constitue la trame de la qualité du tissu social et c'est au sein de sa famille que le mineur en recherche d'autonomie devrait pouvoir intérioriser les règles de la société.

Par ailleurs, cette famille, facteur essentiel de prévention du comportement délinquant, n'échappe pas à son environnement. 
Elle est souvent déstabilisée par les problèmes sociaux causés par les guerres, la pauvreté, l'absence de logements adéquats, l'insuffisance des soins de santé et les difficultés d'accès à une éducation adéquate. Cette absence de satisfaction de besoins fondamentaux constitue souvent un obstacle insurmontable pour que des mineurs puissent accéder, par des moyens légitimes, à la réussite personnelle et sociale. En effet, vivre dans des milieux défavorisés est loin de constituer une condition gagnante pour éviter de succomber à la délinquance.

De façon évidente, les actions à entreprendre dans ce vaste domaine relèvent davantage des États et des organismes voués à la prévention que de la justice, même si celle-ci ne peut pas demeurer indifférente à l'absence de satisfaction des besoins fondamentaux chez l'enfant mineur et chez sa famille qui constituent la cible de ses interventions. II est incontestable que la famille a besoin d'être accompagnée pour assurer sa mission de socialisation de ses membres.

$\mathrm{Si}$, en dépit de cet accompagnement, la famille ne peut assumer sa mission fondamentale, il revient à l'État d'intervenir soit pour protéger la société contre le comportement déviant du mineur, soit pour assurer la protection de l'enfant en danger.

Indépendamment des rôles respectifs qui seront dévolus aux différentes institutions sociales dans la mise en place d'un nouveau modèle d'intervention auprès du mineur, il demeure que leur concertation et leur complémentarité constituent deux piliers indispensables pour assurer la solidité et la stabilité du système.

Il faut se souvenir que le modèle de justice "à la main lourde ", pour employer les termes de McCarney (1999, p. 2), n'a pas eu plus de succès que le modèle de protection dans la réduction de la criminalité juvénile. Bien sûr, la criminalité n'a pas augmenté de façon significative dans nos sociétés. Mais elle n'a pas diminué non plus dans la proportion des sommes affectées aux mesures de répression. II est par ailleurs étonnant de constater que la population semble croire que la criminalité augmente chaque année. Ainsi, même si Statistique Canada rapportait, le 21 juillet 1999, que le taux de criminalité au Canada avait chuté pour une septième année consécutive, atteignant un plancher jamais vu 
depuis près de vingt ans, l'insécurité de la population, elle, a pourtant augmenté (La Presse, 1999, p. A9). De son côté, la criminalité juvénile a diminué ou tout au moins elle n'a pas augmenté, et pourtant la population persiste à croire le contraire. II ne faut donc pas se surprendre que la lutte à la criminalité des mineurs fasse partie maintenant des enjeux électoraux (Hylton, 1994, p. 237). Comment une telle insécurité peut-elle demeurer? Quelles sont les voies de solution à privilégier?

Pour enrayer cette crainte grandissante dans l'opinion publique, nous devons accorder aux victimes des actes délictueux une plus grande place dans nos interventions auprès des mineurs délinquants. C'est le modèle de justice réparatrice actuellement mis en œuvre dans certains pays qui semble être porteur d'espoir pour l'avenir.

II faut s'interroger, en effet, sur la place que la victime occupait dans nos modèles d'intervention antérieurs. II faut bien l'admettre, elle était la plupart du temps reléguée au second rang, quand elle n'était pas tout simplement ignorée ou oubliée.

Cela s'explique par le fait que, dans le modèle de protection, on se préoccupe principalement sinon uniquement de répondre aux besoins spécifiques du mineur délinquant alors considéré davantage comme une victime. Dans ce modèle, la mesure de l'intervention est donc principalement la personne du mineur délinquant. Dans le modèle de justice, on estime que l'infraction a été commise contre la société : le but principal de ce modèle est donc de déterminer la culpabilité de l'accusé et d'imposer une sanction en fonction d'une règle de proportionnalité; les deux principes fondamentaux de l'intervention sont alors la rétribution (le mineur a commis un acte délictueux, il doit en répondre) et la proportionnalité (la sanction sera proportionnelle à la gravité du délit commis).

Pour un modèle de justice réparatrice, l'objectif est d'abord de chercher une façon d'indemniser la victime pour les dommages causés par le mineur délinquant et aussi et surtout de rétablir l'harmonie dans la vie aussi bien de la victime que du mineur délinquant. 
La mise en place d'un tel modèle, on le comprendra, exige que l'on se rapproche davantage des communautés de base et que l'on recherche des solutions qui tiennent vraiment compte de leurs réalités. Elle requiert également la concertation et la complémentarité des divers intervenants (police, services sociaux, services médicaux, écoles, système judiciaire, organismes du milieu, etc.), de même qu'un profond respect des rôles respectifs et des fonctions spécifiques des uns et des autres.

Une telle option doit évidemment s'inscrire à l'intérieur d'une législation qui détermine précisément les responsabilités des différentes catégories d'intervenants. Et cette législation doit maintenir, pour le mineur accusé, la possibilité de demander de saisir la cour, surtout dans les situations où il n'est pas acquis qu'il soit l'auteur de l'infraction.

Il est important d'indiquer que ce modèle de justice réparatrice peut être appliqué aussi bien à l'intérieur du processus judiciaire que dans le cadre de mesures alternatives à la judiciarisation. Dans l'un ou l'autre cas, la victime doit toujours être consentante si elle est invitée à rencontrer le mineur qui est accusé. Quant à ce dernier, il doit préalablement reconnaître sa responsabilité en regard de l'acte délictueux qui a été perpétré ainsi que pour les dommages qu'il a causés.

Ce modèle de justice réparatrice peut être appliqué non seulement dans les situations d'infractions mineures, mais également lorsqu'il s'agit d'infractions majeures, sauf s'il y a un risque de porter atteinte à la sécurité publique ou de miner la confiance du public à l'égard du système judiciaire.

Le facteur déterminant pour s'orienter vers une telle mesure réparatrice est l'assurance que la victime accepte de rencontrer le mineur délinquant. On comprendra que cette rencontre peut permettre à la victime de se rassurer à l'endroit de l'accusé et à ce dernier de se réconcilier éventuellement avec la victime, de fournir à cette dernière des explications sur le geste qu'il a posé et d'assumer une indemnisation appropriée.

Un tel modèle ne garantit évidemment pas à la victime une pleine compensation pour les dommages qu'elle a pu subir. Ce sera 
parfois la société qui supportera une partie des dommages causés; le mineur pourra alors effectuer certains travaux dans la communauté comme dédommagement pour les pertes ainsi occasionnées.

Ce modèle de justice réparatrice fait actuellement l'objet de plusieurs expériences dans différents pays (Omatsu, 1999, p. 22-27). Si l'on ne peut pas encore en tirer des conclusions tout à fait déterminantes, il est d'ores et déjà possible d'affirmer qu'un tel modèle a l'avantage d'apporter une plus grande sécurité aux victimes et d'appliquer des mesures plus adéquates à l'endroit du mineur délinquant. La mise en œuvre de ce modèle de justice réparatrice, en effet, incite le mineur délinquant à accepter la responsabilité de son comportement criminel et à en réaliser les conséquences sur autrui ${ }^{9}$.

II s'agit là, nous semble-t-il, d'une voie nouvelle qui respecte à la fois les acquis du passé et les réalités actuelles de notre monde.

\section{CONCLUSION}

On en conviendra, au cours des trente dernières années le droit de l'enfance et les services offerts aux mineurs ont connu une évolution tout à fait remarquable. On a pris soin notamment de bien distinguer et de dissocier le traitement du mineur en besoin de protection de celui qui commet des infractions. On a également prévu dans les lois des garanties procédurales pour ces deux catégories de jeunes. Au fil des ans, nous avons donc enregistré des acquis incontestables au regard des droits accordés aux mineurs et nous avons précisé à juste titre que le jeune qui commettait des délits ne devait pas être protégé comme une simple victime, mais qu'il devait assumer la responsabilité de ses actes. Nous avons pris davantage conscience de la nécessité de mieux tenir compte des victimes dans les orientations à privilégier. Nous avons aussi réalisé que le traitement du mineur délinquant passait par la concertation et la complémentarité des divers intervenants concernés, de même que par la participation des communautés locales dans le choix et l'application des mesures.

9 Juvenile Justice Models, Australian Law Reform Commission, report no. 84, 1997, p. 476. 
II nous apparaît urgent de nous orienter vers ce nouveau modèle d'intervention. En effet, nous constatons actuellement, dans certains milieux, une tendance à vouloir retourner aux modèles du passé en matière de traitement de la délinquance. Les réformateurs du $19^{\mathrm{e}}$ siècle et du début du $20^{\mathrm{e}}$ siècle avaient obtenu qu'en raison de leur vulnérabilité et de leurs besoins spécifiques les mineurs délinquants soient traités dans un système distinct de celui des adultes. Cet acquis doit demeurer, quel que soit le modèle que nous privilégierons dans l'avenir.

Il est certain que le problème de la délinquance juvénile inquiète au plus haut point la population. Les médias ont souvent tendance à mettre en évidence les situations les plus extrêmes et n'abordent qu'avec parcimonie, quand ils ne les ignorent tout simplement pas, les expériences positives de réhabilitation de mineurs délinquants. Aussi ne faut-il pas se surprendre d'une opinion publique qui s'inquiète et qui exige des lois et des sanctions plus sévères. Nous verrons toujours poindre dans la société à un moment ou à un autre des demandes pour que le système de justice pour mineurs soit de plus en plus répressif.

Faut-il conclure que seule une orientation répressive à l'endroit des mineurs délinquants puisse ouvrir la voie à une paix sociale ou ne risque-t-on pas de détruire alors la fragile qualité du tissu social?

Force nous est de constater que, malgré les acquis des sciences du comportement et des garanties légales, il subsiste encore dans la société des conflits au niveau des options fondamentales entre " la réinsertion sociale ou la sanction, l'assistance ou la punition, la réaction particulière à une situation ou la réaction inspirée par la nécessité de protéger la société dans son ensemble ${ }^{10}$ ".

Avant de nous inscrire dans la voie d'un système de justice plus répressif pour les mineurs, avant en quelque sorte de lancer la serviette, ne pourrions-nous pas nous demander d'abord si, en

\footnotetext{
${ }^{10}$ Voir les commentaires accompagnant l'article 17 des règles minima des Nations Unies concernant l'administration de la justice pour mineurs (Règles de Beijing).
} 
amont de nos interventions, nous avons tout fait pour assurer à chacun et à chacune l'égalité des chances.

N'oublions pas que, si aujourd'hui l'avenir des enfants est entre nos mains, demain ce sera notre avenir qui sera entre les leurs.

Oscar D'AMOURS

Juge à la Cour du Québec

(Chambre de la Jeunesse)

et au Tribunal des droits de la personne

District de Montréal

\section{Références bibliographiques}

A.I.M.J.F. (1998). Chronique, vol. 7, nº 1.

BALA, N. (1994). "The 1995 Young Offenders Act amendments: compromise or confusion », 26 Revue de droit, Université d'Ottawa.

BAllestrem, S. (1997). "La convention des droits de l'enfant et l'administration de la justice des mineurs ", revue Chronique, A.I.M.J.F., vol. 6, $\mathrm{n}^{\circ}$ 1, été.

Cullen, F.T. et K. EgILBERT (1982). Reaffirming Rehabilitation, Criminal Justice Studies, Anderson Publishing (traduction).

EMPEY, L.T., M.C. STAFFORD et C.H. HAY (1999). American Delinquency : Its Meaning and Construction, $4^{\mathrm{e}}$ édition, Belmont, CA, Wadsworth Publishing Co.

FULCHIRON, H. (1997). L'évolution historique de la notion de l'intérêt de l'enfant, une convention, plusieurs regards. Sion, Suisse, Éd. Institut international des droits de l'enfant, octobre.

HACKLER, J. (1991). " Using reintegrative shaming effectively: Why Fiji has a juvenile justice system superior to the U.S., Canada and Australia ", dans J. Hackler (dir.), Official responses to problem juveniles : some international reflexions, Univ. of Alberta, chap. 14. 
HYLTON, J.H. (1994). " Get Tough or Get Smart? Opinions for Canada's Youth Justice System in the Twenty First Century ", Revue canadienne de criminologie, vol. $36, \mathrm{n}^{\circ} 3$.

LA PRESSE (1999). « La criminalité encore en baisse », Montréal, 22 juillet 1999, p. A9.

MCCARNEY, W. (1999). « La criminalité juvénile est-elle hors de contrôle? Mieux vaut prévenir que guérir », revue Chronique, A.I.M.J.F., vol. 8, $n^{\circ}$ 1 , juillet.

MCCARNEY, W. (1997). "Les instruments des Nations Unies concernant la délinquance juvénile », revue Chronique, A.I.M.J.F., vol. 6, nº 1, été.

OMATSU, M. (1999). "Experiences with Restorative Justice », Journal des juges provinciaux, vol. 23, $\mathrm{n}^{\circ}$ 1, printemps.

TRÉPANIER, J. (1989). "Principes et objectifs guidant le choix des mesures prises en vertu de la Loi sur les jeunes contrevenants », Revue du Barreau, Québec, vol. 49.

TRÉPANIER, J. et F. TULKENS (1995). Délinquance et protection de la jeunesse, PUM, P.U.O. et De Boeck Université.

TULKENS, F. (1996). Revue générale de droit.

WINTERDYK, J.A. (dir.) (1997). Juvenile Justice Systems, International Perspectives, Toronto, Canadian Scholars Press.

ZeRmatTen, J. (1994). Revue internationale de criminologie et de police technique, vol. 2. 